\title{
Gases bosônicos armadilhados
}

\author{
(Trapped Bose gases)
}

\author{
A.G. de Sousa ${ }^{1}$ e V.S. Bagnato ${ }^{2}$ \\ ${ }^{1}$ Universidade de São Paulo, Instituto de Química de São Carlos, Departamento de Química \\ e Física Molecular, São Carlos, São Paulo, Brasil. \\ ${ }^{2}$ Universidade de São Paulo, Instituto de Física de São Carlos, São Carlos, São Paulo, Brasil.
}

\begin{abstract}
O espectro dos modos coerentes para gás atômico de Bose diluído, confinado em uma armadilha harmônica não isotrópica é determinado e utiliza-se da teoria de perturbação otimizada e a técnica das raízes aproximantes auto-similares. As expressões obtidas são válidas para níveis de energia arbitrários de todos os modos coerentes excitados. Este artigo deve ser utilizado como ponto de início para os alunos que querem se aprofundar no tema de condensação de Bose-Einstein atualmente presente em quase todos periódicos de Física.
\end{abstract}

Palavras-chave: espectro do níveis de energia, gases bôsonicos armadilhados, teoria de perturbação otimizada.

The spectrum of coherent modes for dilute atomic Bose gas, confined in a non-isotropic harmonic trap, is determined by using the optimized perturbation theory and the technique of self-similar root approximants. The obtained expressions are valid for arbitrary energy levels of all excited nonlinear coherent modes. This article must be used as an introduction for the students that want to deepen nowadays in the theme of the Bose-Einstein condensation.

Keywords: Bose trapped gases, energy spectrum, optimezed perturbation theory.

\section{I Introdução}

Desde sua realização experimental a condensação de Bose-Einstein tem sido tema constante na maioria dos periódicos internacionais. Desta forma, é interessante fornecer subsídios para que os estudantes do curso de pós-graduação possam acompanhar tal vasta literatura.

Em baixas temperaturas, o condensado Bose-Einstein de gases atômicos confinados é bem descrito pela equação de Gross-Pitaevskii [1-3]. Em equilíbrio, o estado condensado corresponde à solução do estado fundamental desta equação. A estrutura matemática da equação de GrossPitaevskii é idêntica à equação de Schrödinger não linear. Na presença de um potencial de confinamento, ela possuirá um conjunto discreto de estados estacionários. Tais estados podem ser interpretados como modos coerentes não lineares de átomos confinados [4] correspondendo ao condensado de Bose no estado não fundamental. As excitações destes modos podem ser realizadas por meio da modulação ressonante de um potencial externo [4-6]. Tais modos têm sido estudados em Refs. [7-9] e o modo dipolo tem sido observado experimentalmente [10].

Não devemos confundir os modos coerentes não lineares, que são soluções estacionárias da equação de Gross-Pitaevskii não linear, com excitações coletivas elementares, que são soluções das equações de Bogolubov-De Gennes lineares [3]. Os modos coerentes não lineares são às vezes chamados de modos topológicos. Cada modo não linear produz suas próprias excitações coletivas como pequeno desvio em volta do modo dado. Estas excitações

Enviar correspondência para A.G. de Sousa. E-mail: gama@iqsc.usp.br. coletivas correspondem às pequenas flutuações de densidades e estão relacionados com o espectro de Bogolubov para o hélio superfluido [11] e o condensado Bose-Einstein confinado [12]. No primeiro caso, está o espectro fônon-roton (hélio superfluido) $[13,14]$. No caso dos átomos confinados diluídos, é um ramo fônon-partícula (condensado Bose-Einstein confinado). As excitações coletivas em volta dos modos coerentes do estado não fundamental ainda não foram consideradas.

Antes de considerar as perturbações dos modos coerentes não lineares, é necessário ter uma descrição precisa das suas propriedades. Em particular, para melhor entender as características do espectro dos modos não lineares. Os estudos deste espectro foram iniciados na Ref. [4] e no caso de uma interação fraca, foi também considerado na Ref. [9]. No presente trabalho, daremos uma descrição geral do espectro de modo não linear para átomos confinados em uma armadilha cilíndrica. Nós descreveremos um método para calcular os níveis de energia arbitrários deste espectro para alguma magnitude do parâmetro de interação. Também construiremos expressões analíticas aproximadas para o espectro. Tendo em mãos tais expressões analíticas é conveniente estudar o espectro dependente dos números quânticos e parâmetros do sistema com resultados experimentais.

\section{Equação para modos coerentes}

As interações de átomos em gases diluídas são caracterizadas pelo potencial de contato de Fermi 


$$
\Phi(r)=A \delta(r), \quad A \equiv 4 \pi \eta^{2} \frac{a_{s}}{m_{0}},
$$

no qual $a_{s}$ é o comprimento de espalhamento da onda-s $m_{0}$, a massa atômica e $A$ representa a interação de cada partícula. O potencial de confinamento é geralmente tomado na forma

$$
U(\mathbf{r})=\frac{m_{0}}{2}\left(\omega_{x}^{2} r_{x}^{2}+\omega_{y}^{2} r_{y}^{2}+\omega_{z}^{2} r_{z}^{2}\right),
$$

onde $\omega_{x}, \omega_{y}$ e $\omega_{z}$ são as freqüiências do oscilador.

O campo coerente $\varphi(\mathbf{r})$, normalizado para unidade, é descrito [3] pela equação

$$
\mathbf{H}[\varphi(\mathbf{r})] \varphi(\mathbf{r})=\mathrm{E} \varphi(\mathbf{r}),
$$

o qual é freqüientemente chamado da equação de GrossPitaevskii, com Hamiltoniano não linear

$$
\mathbf{H}[\varphi(\mathbf{r})]=-\frac{\eta^{2} \nabla^{2}}{2 m_{0}}+U(r)+N A|\varphi(r)|^{2},
$$

onde $N$ é o número de partículas condensadas.

Por causa do potencial de confinamento $U(\mathbf{r})$, o autovalor (1) possui o espectro discreto, então as autofunções relacionadas são os modos coerentes não lineares [4].

Nós consideraremos um potencial harmônico de simetria cilíndrica, com uma freqüência radial

$$
\omega_{r} \equiv \omega_{x}=\omega_{y},
$$

e o parâmetro anisotrópico

$$
v \equiv \frac{\omega_{z}}{\omega_{r}}
$$

define a assimetria do confinamento.

É conveniente trabalhar com quantidades adimensionais, medindo as variáveis espaciais

$$
r \equiv \frac{\sqrt{r_{x}^{2}+r_{y}^{2}}}{l_{r}}, \quad z \equiv \frac{r_{z}}{l_{r}},
$$

onde o comprimento do oscilador transversal é definido como

$$
l_{r} \equiv \sqrt{\frac{\eta}{m_{0} \omega_{r}}} .
$$

O parâmetro de acoplamento $(g)$ é definido como

$$
g \equiv 4 \pi \frac{a_{s}}{l_{r}} N
$$

sendo uma quantidade adimensional caracterizando interações atômicas. Definindo o Hamiltoniano e a função de adimensional

$$
\mathbf{H} \equiv \frac{H[\varphi(r)]}{\eta \omega_{r}}, \quad \psi(x, y, z) \equiv l_{r}^{3 / 2} \varphi(r) .
$$

Em coordenadas cilíndricas, temos

$$
\mathrm{H}=-\frac{1}{2} \nabla^{2}+\frac{1}{2}\left(r^{2}+v^{2} z^{2}\right)+g|\psi|^{2},
$$

onde

$$
\nabla^{2}=\frac{\partial^{2}}{\partial r^{2}}+\frac{1}{r} \frac{\partial}{\partial r}+\frac{1}{r^{2}} \frac{\partial^{2}}{\partial \varphi^{2}}+\frac{\partial^{2}}{\partial z^{2}} .
$$

Então o autoproblema (1) transforma à equação

$$
\mathrm{H} \Psi_{n}=\mathrm{E}_{n} \Psi_{n} \text {. }
$$

Definindo os modos coerentes adimensionais $\Psi_{n} \mathrm{e}$ seu espectro $\left\{E_{n}\right\} ; \mathrm{n}$ sendo um multi-índice dos modos. No final são assumidos para ser normalizados como

$$
\int_{0}^{\infty} r d r \int_{0}^{2 \pi} d \varphi \int_{-\infty}^{\infty} d z\left|\Psi_{n}(r, \varphi, z)\right|^{2}=1 .
$$

Podemos encontrar o espectro $\left\{E_{n}\right\}$ para todos os níveis de energia $E_{n}$ relacionados com índice quântico $n$ e parâmetros de acoplamento arbitrário e anisotrópico $g$ e $v$, respectivamente.

\section{Teoria de perturbação otimizada}

Para calcular o espectro do autovalor (10), nós empregaremos a teoria de perturbação otimizada, que consiste na análise numérica do espectro estudado [15-19], o qual tem sido aplicado por um número de modelos em Mecânica Quântica, Física Estatística e Teoria Quântica de Campo [15-26]. Numerosa referência em várias aplicações pode ser encontrada em revistas [27-29]. É importante enfatizar que a teoria de perturbação otimizada foi mostrada para fornecer resultados precisos para todos os níveis de energia e parâmetros de acoplamento grandes.

Começamos com Hamiltoniano inicial de um oscilador harmônico

$$
\mathrm{H}=-\frac{1}{2} \nabla^{2}+\frac{1}{2}\left(u^{2} r^{2}+v^{2} z^{2}\right),
$$

tendo o oscilador os comprimentos como dois parâmetros de teste, $u$ e $v$. Este Hamiltoniano possue os autovalores

$$
\mathrm{E}_{n m j}^{(0)}=(2 n+|m|+1) u+\left(j+\frac{1}{2}\right) \varphi,
$$

Com o número quântico radial $n=0,1,2, \ldots$, número azimutal $m=0, \pm 1, \pm 2$, número quântico axial $j=0,1,2 \ldots$ A função de onda correspondente é

$$
\begin{aligned}
& \Psi_{n m j}^{(0)}(r, \varphi, z)=\left[\frac{2 n ! u^{|m|+1}}{n+|m|}\right] r^{|m|} e^{\left(-\frac{u}{2} r^{2}\right)} \times \\
& L_{n}^{|m|}\left(u r^{2}\right) \frac{e^{i m \varphi}}{\sqrt{2 \pi}}\left(\frac{v}{\pi}\right) \frac{1}{\sqrt{2^{j} j !}} e^{\left(-\frac{v}{2} z^{2}\right)} \mathrm{H}_{j}(\sqrt{v} z),
\end{aligned}
$$

onde $L_{n}^{m}$ (.) é um polinômio de Laguerre e $H_{j}$ é um polinômio de Hermite.

Usando um variante da teoria de perturbação padrão, tal com teoria de Rayleigh-Schröndiger, nos permite achar uma seqüência $\left\{E_{k}(g, u, v)\right\}$ das energias $E_{k}(g, u, v)$ para 
ordens de aproximação $k=0,1,2, \ldots$ Aqui, por simplicidade de notação, as dependências dos níveis de energia nos números quânticos não são escritas abaixo explicitamente, mas são assumidos. Por exemplo, $E_{0}(g, u, v)=\mathrm{E}_{n m j}^{(0)}$. Então os parâmetros de teste serão transformados em funções de controle[15-19] $u_{k}(g)$ e $v_{k}(g)$ tais que apresentam a seqüência $\left\{e_{k}(g)\right\}$ dos termos otimizados

$$
\mathrm{e}_{\mathrm{k}}(g) \equiv \mathrm{E}_{k}\left(g, u_{k}(g), v_{k}(g)\right)
$$

Convergente. As funções controle podem ser encontradas das condições de otimização. Por exemplo, nós podemos empregar a condição do ponto-fixo

$$
\left(\delta u \frac{\partial}{\partial u}+\delta v \frac{\partial}{\partial v}\right) \mathrm{E}_{k}(g, u, v)
$$

cujas soluções são $u=u_{k}(g)$ e $v=v_{k}(g)$. Os aproximantes otimizados (14) são válidos para números quânticos arbitrários e para o parâmetro de acoplamento $g$. O mesmo procedimento pode ser usado para armadilhas anarmônicas, descritos pelo potencial de confinamento anarmônico de potências diferentes, inteiros ou não inteiros. Note que o uso de armadilhas anarmônicas pode ser importante para alguns experimentos [30] tratando com excitações coletivas elementares.

Em primeira ordem, temos a energia

$$
\mathrm{E}_{1}(g, u, v)=\frac{p}{2}\left(u+\frac{1}{u}\right)+\frac{q}{4}\left(v+\frac{v^{2}}{v}\right)+u \sqrt{v} g I_{n m j} .
$$

Os números quânticos são introduzidos através das combinações

$$
p \equiv 2 n+|m|+1, \quad q \equiv 2 j+1
$$

E da integral

$$
\begin{aligned}
& \mathrm{I}_{n m j}=\frac{1}{u \sqrt{v}} \int\left|\Psi_{n m j}^{(0)}(r, \varphi, z)\right|^{4} r d r d \varphi d z=\frac{2}{\pi^{2}}\left[\frac{n !}{(n+|m|) 2^{j} j !}\right] \times \\
& \int_{0}^{\infty} x^{2|m|} e^{-2 x}\left[L_{n}^{|m|}(x)\right] d x \int_{0}^{\infty} e^{-2 t^{2}} \mathrm{H}_{j}^{4}(t) d t .
\end{aligned}
$$

A condição do ponto-fixo (15) produz as equações

$$
p\left(1-\frac{1}{u^{2}}\right)+\frac{s}{p v} \sqrt{\frac{v}{q}}=0, q\left(1-\frac{v^{2}}{v^{2}}\right)+\frac{u s}{p v \sqrt{v q}}=0,
$$

para funções controle $u=u_{1}(g)$ e $v=v_{1}(g)$, onde, por conveniência, a notação

$$
s \equiv 2 p \sqrt{q} g \vee \mathrm{I}_{n m j}
$$

é usada. Substituindo as soluções para as funções controle, dadas pelas Eqs. (18), na forma (16) resulta no aproximante otimizado $e_{1}(g)$, de acordo com a definição (14). Este procedimento continua para uma desejada ordem de aproximação. Aqui limitamos na aproximação de primeira ordem

$$
\mathrm{E} \equiv e_{1}(g)=\mathrm{E}_{1}\left(g, u_{1}(g), v_{1}(g)\right),
$$

em que as funções de controle $u_{1}(g)$ e $v_{1}(g)$ são soluções das Eqs. (18). As equações finais, para um dado conjunto $\{n, m$, j\} dos números quânticos e um parâmetro de acoplamento exige uma solução numérica. Portanto, o espectro (20) pode ser calculado apenas numericamente. Entretanto, é sempre desejável possuir uma expressão analítica aproximada que poderia ser mais fácil tratar com respeito à variação dos parâmetros do sistema, tais como: números quânticos e o parâmetro de acoplamento.

\section{Raízes aproximantes auto-similares}

Este método permite reconstruir expressões analíticas do espectro dos modos coerentes não lineares [31,32]. Então, precisamos conhecer as expansões assintóticas do espectro nos limites dos acoplamentos fraco e forte. Estas expansões para o espectro (20) podem ser derivadas das Eqs. (16) e (18). É conveniente usar a variável (19) que é proporcional ao parâmetro de acoplamento; assim se $g \rightarrow 0$, então $s \rightarrow 0$, e quando $g \rightarrow \infty$, então $s \rightarrow \infty$. No limite de acoplamento-fraco, nós encontramos a expansão

$$
\mathrm{E} \approx a_{0}+a_{1} s+a_{2} s^{2}+a_{3} s^{3} .
$$

Para acoplamento fraco podemos fazer $s \rightarrow 0$. Os coeficientes $a_{i}$ podem ser obtidos [32] onde

$$
a_{0}=p+\frac{q v}{2}, \quad a_{1}=\frac{1}{2 p\left(q v^{1 / 2}\right)}, a_{3}=\frac{(p+2 q v)^{2}}{16 p^{5}(q v)^{7 / 2}}
$$

No limite de acoplamento forte, obtemos

$\mathrm{E} \approx b_{0} s^{2 / 5}+b_{1} s^{-2 / 5}+b_{2} s^{-6 / 5}+b_{3} s^{-2}+b_{4} s^{-18 / 5}$,

onde

$$
\begin{aligned}
& 4 b_{0}=5, \quad 4 b_{1}=2 p^{2}+(q v)^{2} \\
& 20 b_{2}=-3 p^{4}+2 p^{2}(q v)^{2}-2(q v)^{4} \\
& 20 b_{3}=2 p^{6}-p^{4}(q v)^{2}-2 p^{2}(q v)^{4}+2(q v)^{6} \\
& 500 b_{4}=-44 p^{8}+22 p^{6}(q v)^{2}+2 p^{4}(q v)^{4} \\
& +78 p^{2}(q v)^{6}-69(q v)^{8} \\
& \begin{array}{r}
12500 b_{5}=1122 p^{10}-595 p^{8}(q v)^{2}-70 p^{6}(q v)^{4} \\
+440 p^{4}(q v)^{6}-3640(q v)^{8}+2821(q v)^{10}
\end{array}
\end{aligned}
$$

As expansões assintóticas (21) e (22), válidos no limite de acoplamento-fraco $s \rightarrow 0$ e no limite de acoplamento-forte $s \rightarrow \infty$, respectivamente, podem ser construídas usando a técnica dos aproximantes cruzados auto-similares [31,32]. Esta técnica constrói fórmulas interpolativas que fornecem corretas expansões assintóticas, resultando em uma boa precisão no intervalo inteiro da variável $s$. A descrição detalhada do método pode ser encontrada nas Refs. [31,32]. Com apenas um termo na expansão de acoplamento-forte (22), nós obtemos a raiz aproximante auto-similar de primeira ordem

$$
\mathrm{E}_{1}^{*}=a_{0}\left(1+C_{0} s\right)^{2 / 5},
$$

no qual 


$$
a_{0}=p+\frac{q v}{2}, \quad C_{0} a_{0}^{2 / 5}=1,746928 .
$$

Retendo dois termos na Eq. (22) fornece o aproximante de segunda ordem

$$
\mathrm{E}_{2}^{*}=a_{0}\left[\left(1+C_{1} s\right)^{6 / 5}+C_{2} s^{2}\right]^{1 / 5},
$$

onde $a_{0}$ é o mesmo da Eq. (23) e

$$
C_{1} a_{0}^{25 / 6}=2,533913\left[2 p^{2}+(q v)^{2}\right]^{5 / 6}, C_{2} a_{0}^{5}=3,051758 .
$$

Similarmente, encontramos o de terceira ordem

$\mathrm{E}_{3}^{*}=a_{0}\left\{\left[\left(1+B_{1} s\right)^{6 / 5}+B_{2} s^{2}\right]^{11 / 10}+B_{3} s^{3}\right\}^{2 / 15}$,

onde $a_{0}$ é novamente o mesmo da Eq. (23) e

$$
\begin{gathered}
B_{1} a_{0}^{125 / 22}\left[2 p^{2}+(q v)^{2}\right]^{5 / 66}= \\
1,405455\left[8 p^{4}+12 p^{2}+(q v)^{2}+(q v)^{4}\right]^{5 / 6} \\
B_{2} a_{0}^{75 / 11}=6,619620\left[2 p^{2}+(q v)^{2}\right]^{10 / 11} \\
B_{3} a_{0}^{15 / 2}=5,331202 .
\end{gathered}
$$

No mesmo caminho, nós podemos construir aproximantes de alta ordem, que não escreveremos abaixo explicitamente.

Deixa-nos enfatizar que as raízes aproximantes autosimilares (23) à (25) são válidas para toda extensão da variável (19). As precisões das raízes aproximantes podem ser avaliadas por comparar os seus valores com aqueles obtidos numericamente das Eqs. (18) e (20).

O condensado Bose-Einstein é freqüentemente tratado por usar a aproximação de Thomas-Fermi (TF), quando se omite o operador energia cinética na Eq. (10). Em tal caso, a solução para Eq. (10), torna-se aproximação (TF)

$$
\Psi_{T F}(r, z)=\Theta\left(r_{0}^{2}-r^{2}-v^{2} z^{2}\right)\left(\frac{r_{0}^{2}-r^{2}-v^{2} z^{2}}{2 g}\right)^{1 / 2},
$$

onde $r_{0}^{2} \equiv 2 \mathrm{E}_{T F}$ e a energia é obtida da condição de normalização para $\Psi_{T F}$, que resulta na expressão

$$
\mathrm{E}_{T F}=\frac{1}{2}\left(\frac{15}{4 \pi} g v\right)^{2 / 5} \text {. }
$$

A aproximação de Thomas-Fermi não é auto-consistente desde que a média do Hamiltoniano (9), calculado com a função de onda $\Psi_{T F}$, diverge. A energia $E_{T F}$ é válida para um parâmetro de acoplamento assintoticamente grande $\mathrm{g} \rightarrow \infty$, mas para $g$ pequeno e intermediário esta fórmula não é precisa. Várias sugestões foram propostas para melhorar a aproximação de Thomas-Fermi por meio de correções adicionais [33-35]. Entretanto, esta aproximação fornece-nos unicamente a energia do estado fundamental, sendo incapaz para descrever os níveis de energia excitados dos modos coerentes não lineares.

\section{Conclusão}

Os modos coerentes não lineares do condensado Bose-Einstein na temperatura zero foram estudados. Estes modos coerentes são descritos pela equação de GrossPitaevskii e correspondem as soluções estacionárias desta equação. Na presença de um potencial de confinamento, o espectro dos modos coerentes é discreto. Então encontramos os níveis de energia deste espectro relacionados com os números quânticos arbitrários e alguns valores dos parâmetros de acoplamento e outras propriedades diferentes utilizadas em uma armadilha cilíndrica. Calculamos o espectro dos níveis de energia através da teoria de perturbação otimizada, o qual exigiu a solução numérica das equações através das funções de controle. Outro método empregado foi das raízes aproximantes auto-similares, que resultaram nas expressões analíticas para o espectro dos níveis de energia. A vantagem de se obter expressões analíticas está na viabilidade de um estudo mais detalhado do comportamento de todos os parâmetros que caracterizam o sistema.As propriedades dos modos coerentes estão sendo melhores compreendidas através das condições de otimização através da observação experimental.

\section{Agradecimentos}

Este trabalho foi realizado no Centro de Pesquisa de Óptica, Universidade de São Paulo, São Carlos. Agradecemos ao Prof. Dr. V.I. Yukalov pela colaboração nos cálculos e dos métodos utilizados, ao Prof. Dr. Albérico Borges F. da Silva pela cooperação e também o suporte financeiro dado pela CAPES.

\section{Referências}

[1] A.S. Parkins and D.F. Walls, Phys. Rep. 303, 1 (1998).

[2] F. Dalfovo, S. Giorgini, L.P. Pitaevskii, and S. Stringari, Rev. Mod. Phys. 71, 463 (1999).

[3] P.W. Courteille, V.S. Bagnato and V.I. Yukalov, Laser Phys. 11, 659 (2001).

[4] V.I. Yukalov, E.P. Yukalova and V.S. Bagnato, Phys. Rev. A 56, 4845 (1997).

[5] V.I. Yukalov, E.P. Yukalova and V.S. Bagnato, Laser Phys. 10, 26 (2000).

[6] V.I. Yukalov, E.P. Yukalova and V.S. Bagnato, Laser Phys. 11, 455 (2001).

[7] E.A. Ostrovskaya, et al., Phys. Rev. A 61, 031601 (2000).

[8] D.L. Feder, et al., Phys. Rev. A 62, 053606 (2000).

[9] Y.S. Kivshar, T.J. Alexander, and S.K. Turitsyn, Phys. Lett. A 278, 225 (2001).

[10] J.E. Williams, et al., Phys. Rev. A 61, 33612 (2000).

[11] A.D.B. Woods and R.A. Cowley, Rep. Prog. Phys. 36, 1135 (1973).

[12] J. Steinhauer, R. Ozeri, N. Katz and N. Davidson, Phys. Rev. Lett. 88, 120407 (2001).

[13] Y.A. Nepomnyashchy, Phys. Rev. B 46, 6611 (1992).

[14] E.C. Svensson, W. Montfrooj and I.M. de Schepper, Phys. Rev. Lett. 77, 4398 (1996).

[15] V.I. Yukalov, Mosc. Univ. Phys. Bull. 31, 10 (1976).

[16] V.I. Yukalov, Theor. Math. Phys. 28, 652 (1976).

[17] V.I. Yukalov, Physica A 89, 363 (1977).

[18] V.I. Yukalov, Ann. Physik 36,31 (1979).

[19] V.I. Yukalov, Ann. Physik 37, 171 (1980). 
[20] W.E. Caswell, Ann. Phys. 123, 153 (1979).

[21] I. Halliday and P. Suranyi, Phys. Rev. D 21, 1529 (1980).

[22] P.M. Stevenson, Phys. Rev. D 23, 2916 (1981).

[23] J. Killingbeck, J.Phys. A 14, 1005 (1981).

[24] F.M. Fernandez and E.A. Castro, Phys. Lett. A 88, 4 (1982).

[25] I.D. Feranchuk and Komarov, L.I., Phys. Lett. A 88, 211 (1982).

[26] A. Okopinska, Phys. Rev. D 35, 1835 (1987).

[27] M. Dineykan, G.V. Efimov, G. Gandbold and S.N. Nedelko, Oscilator Representation in Quantum Physics (Springer, Berlin, 1995).
[28] A.N. Sissakian and I.L Solovtsov, Phys. Part. Nucl. 30, 1057 (1999).

[29] V.I. Yukalov and E.P. Yukalova, Ann. Phys. 277, 219 (1999).

[30] K.P. Marzlin and W. Zhang, Phys. Lett. A 248, 290 (1998).

[31] V.I. Yukalov, E.P. Yukalova and S. Gluzman, Phys. Rev. A 58, 96 (1998).

[32] S. Gluzman and V.I. Yukalov, Phys. Rev. E 58, 4197 (1998).

[33] F. Dalfovo, L. Pitaevskii and S. Stringari, Phys. Rev. A 54, 4213 (1996).

[34] E. Lundth, C.J. Pethick and H. Smith, Phys. Rev. A 55, 2126 (1997).

[35] A.L. Fetter and D.L. Feder, Phys. Rev. A 58, 3185 (1998). 